\title{
Langue de spécialité : cohésion, culture et cohérence
}

Une approche discursive

Shirley Carter-Thomas

\section{OpenEdition}

\section{Journals}

Édition électronique

URL : http://journals.openedition.org/asp/4019

DOI : 10.4000/asp.4019

ISSN : 2108-6354

Éditeur

Groupe d'étude et de recherche en anglais de spécialité

Édition imprimée

Date de publication : 1 décembre 1994

Pagination : 61-67

ISSN : 1246-8185

Référence électronique

Shirley Carter-Thomas, "Langue de spécialité : cohésion, culture et cohérence », ASp [En ligne], 5-6 | 1994, mis en ligne le 28 novembre 2013, consulté le 19 avril 2019. URL : http:// journals.openedition.org/asp/4019; DOI : 10.4000/asp.4019

Ce document a été généré automatiquement le 19 avril 2019

Tous droits réservés 


\section{Langue de spécialité : cohésion, culture et cohérence}

Une approche discursive

Shirley Carter-Thomas

1 Données culturelles et éléments linguistiques propres sont étroitement imbriqués dans la production et l'interprétation de tout texte. Un texte de spécialité tel qu'un article scientifique est solidement ancré dans une réalité technique et culturelle spécifiques. Il véhicule un savoir propre au champ scientifique en question et son élaboration linguistique ne peut que refléter cette spécificité, car si nous admettons que l'usage d'une langue est primordialement une activité sociale, il s'ensuit que le discours scientifique, comme tout autre acte de communication, reste fortement déterminé par les pratiques sociales et culturelles qui l'engendrent et en même temps le contraignent. Vue sous cet angle, la notion de genre est, par essence, culturelle.

2 Le texte de spécialité est conçu pour être lu. Selon le genre de texte, le lecteur aura des attentes différentes qui influenceront son interprétation. Nous nous adaptons au type de texte auquel nous sommes confrontés. Tout comme, en tant que professeurs et linguistes, nous nous adaptons aux écrits de nos élèves qui rédigent dans une langue seconde.

3 Depuis plusieurs années, je corrige et évalue les projets scientifiques des élèves ingénieurs à l'Institut National des Télécommunications (INT). Ces projets font partie de leur cursus en langues en troisième année. La consigne est la suivante : rédiger en anglais une synthèse de 10 pages sur un sujet relatif au domaine des télécommunications. Le texte doit être accessible à un public scientifique général, c'est-à-dire non spécialiste du domaine. Ce travail fait l'objet d'une double correction. Le professeur de langues donne une première note qui concerne non seulement la correction de la langue (temps verbaux, articles, prépositions, orthographe), mais également l'organisation et l'enchaînement logique du discours ainsi que la maîtrise du registre. La deuxième note est donnée par un enseignant spécialiste du domaine qui évalue exclusivement le contenu scientifique. 
4 À partir de cette expérience, je me propose d'examiner les facteurs qui entrent en jeu dans notre interprétation et notre évaluation de ce travail. Quels sont les éléments qui contribuent à l'interprétation de la cohérence d'un texte?

Dans le cadre d'une analyse textuelle, la question fondamentale, pour tout professeur de langue et linguiste, est de comprendre ce qui fait qu'une suite de phrases devient un texte: c'est la mise en relation des caractéristiques de surface d'une part et d'un jugement plus subjectif sur la qualité et la lisibilité de l'ensemble d'autre part. Autrement dit, il s'agit des liens entre les notions de cohésion et cohérence.

\section{Cohésion}

6 Tout ce qui concerne la cohésion d'un texte reste relativement objectif et quantifiable. Le texte devient, en quelque sorte, l'objet statique d'une analyse formelle. C'est ainsi que D. Slatka (1975: 31) a proposé une définition des notions de cohésion et cohérence, fondée sur une distinction rigoureuse entre « texte » et « discours ».

Plan de la signifiance (système de règles linguistiques formelles)

Texte <-----> phrase <-----> morphème

Plan de la signification (ensemble de normes sociales concrètes)

Discours <-----> énoncé <-----> mot

7 Il distingue le texte, " objet formel abstrait », du discours, "pratique sociale concrète ». L'analyse de la cohésion reste ainsi une analyse (théoriquement) purement linguistique. Il s'agit du fonctionnement interne du texte qui ne fait pas intervenir les facteurs relevant de la situation d'énonciation ou d'ordre pragmatique.

Dans le contexte de l'apprentissage d'une langue étrangère, l'analyse des caractéristiques de surface portera évidemment sur les fautes provenant d'une maîtrise imparfaite du système linguistique. L'analyse de la cohésion nous permettra d'apprécier la maitrise des règles linguistiques de base, dans la mesure où c'est l'emploi correct des procédés morpho-syntaxiques et sémantiques qui permet de dégager les relations à l'intérieur de la phrase, et également les liens entre les phrases qui constituent le texte.

9 Parmi les linguistes qui ont cherché à cerner la notion de cohésion, M.A.K. Halliday et R. Hassan sont devenus une référence incontournable. Dans leur ouvrage intitulé Cohesion in English (1976), ils font l'inventaire des procédures linguistiques à la disposition du scripteur pour faire «coaguler» son texte qu'ils classent sous cinq grandes rubriques : référence, substitution, ellipse, conjonctions et cohésion lexicale.

10 Sous la rubrique "référence », par exemple, figurent les articles définis et indéfinis, les pronoms personnels, les démonstratifs et tout ce qui peut jouer un rôle anaphorique ou cataphorique dans le texte et ainsi contribuer à sa cohésion interne. Les éléments exophoriques (dont le référent se situe en dehors du texte dans la situation d'énonciation) ne sont pas, selon les auteurs, « cohésifs » à proprement parler, car ils ne contribuent pas à cette cohésion interne. Cependant, cette distinction n'est pas toujours facile à opérer, notamment dans le cas de la cohésion lexicale.

11 Sous cette rubrique, les auteurs distinguent deux sous-catégories: la réitération et l'association (collocation). Ainsi, entre computer et keyboard, il y a, pour la plupart des gens, une association sémantique, ce qui pourrait créer, si on les trouvait ensemble dans un texte, un lien de cohésion. Toutefois, ce qui constitue un champ associatif dépend dans 
une large mesure des connaissances extra-linguistiques communes aux participants de l'acte de communication. Les données culturelles ne peuvent qu'y être associées.

La question des connaissances partagées (ou supposées telles) se trouve au premier plan dans l'analyse d'un texte écrit. Si, à l'oral, une réadaptation au savoir de l'auditoire peut se faire au moyen d'apport de précisions et de reformulations, à l'écrit, le niveau d'accessibilité doit être fixé dès le départ. Dans un discours scientifique, où la terminologie est abondante, l'importance de l'adaptation aux connaissances de l'autre reste primordiale. Les scripteurs se voient dans l'obligation de faire usage d'un grand nombre de termes spécifiques, d'anaphoriques très variés qui renvoient à la même notion. Cependant, si ses connaissances extra-linguistiques dans le domaine sont insuffisantes, le lecteur ne saura pas établir le lien de cohésion, par exemple, entre deux synonymes,

Si l'analyse de la cohésion d'un texte donné ne se délimite pas facilement, c'est justement parce que les deux concepts, cohésion et cohérence, restent largement interdépendants. Un texte qui est très cohésif, qui possède une structure interne où les liens de cohésion sont facilement repérables, fournit au lecteur des outils précieux facilitant l'interprétation. La cohésion n'est pas cependant une fin en soi. Tout professeur sait intuitivement que la quantité des corrections ayant trait à la surface textuelle n'est pas forcément en proportion directe avec la qualité ou l'efficacité du texte en tant qu'ensemble. La présence de marques de cohésion ne garantit pas qu'un texte sera perçu comme cohérent. La cohésion textuelle n'est que l'un des éléments contribuant à l'interprétation de la cohérence.

\section{Cohérence}

14 Afin d'étudier les rapports entre les concepts de cohésion et de cohérence, il est nécessaire de replacer le texte dans son contexte discursif. Nous pouvons définir le discours comme étant une unité plus large que le texte "qui n'exclut pas la prise en compte des manifestations formelles de l'organisation interphrastique, mais nécessite également la prise en considération de facteurs situationnels et interpersonnels » (Fernandez 1984).

15 Face à une suite de phrases peu claires et désordonnées, le lecteur essaiera de faire intervenir ses connaissances, non seulement sur le contexte linguistique immédiat, mais également sur la situation d'énonciation et ses connaissances du monde extérieur au texte afin de réinterpréter et d'établir une cohérence. Il peut se servir de marques formelles de cohésion présentes dans le texte pour l'aider dans sa tâche, mais ce faisant, il présuppose au départ une cohérence au discours. Selon Michel Charolles (1978 : 38) :

... tout se passe comme si le « récepteur ignorant » faisait crédit de cohérence à

l'émetteur, admettait qu'il a ses raisons et s'efforçait de les retrouver afin de reconstruire le suivi de son discours.

Cette présupposition au départ d'une cohérence du discours occupe une place toute particulière pour le professeur de langue devant un texte scientifique spécialisé. Le présupposé bienveillant du lecteur ne risque-t-il pas de l'amener à rétablir une cohérence au départ inexistante sous couvert de "technicité » du texte, alors qu'il n'y avait qu'incompréhension des sources par l'élève? Dans le contexte du projet scientifique des élèves de l'INT, il s'agit d'une synthèse de textes préexistants dans le domaine en question. Comment peut-on mesurer leur compréhension des textes sources? Dans les 
cas litigieux, une incompréhension totale sera vite mise en évidence lors de la soutenance orale (qui suit la remise du document écrit) en présence du professeur de langue et de l'enseignant scientifique. Cependant, dans la plupart des cas, une imparfaite maitrise des textes sources donnera lieu à un collage de lambeaux de textes incohésifs en eux-mêmes.

En tant qu'enseignants, notre rôle n'est pas seulement celui du lecteur intéressé, mais également celui de l'évaluateur. L'élève rédacteur est conscient du fait qu'il écrit pour être évalué, d'où, peut-être, une accumulation de marqueurs explicites de continuité destinés à réduire les efforts de réinterprétation et à guider le lecteur de façon claire vers une interprétation de la cohérence.

$18 \mathrm{Si}$, toutefois, la présence de liens explicites de cohésion n'est pas suffisante pour garantir la cohérence d'un texte, quels sont les autres marqueurs qui peuvent également contribuer à notre interprétation de la cohérence ? Nous avons déjà soulevé l'importance de la notion de genre, à laquelle nous reviendrons. Cependant, il existe un autre aspect qui reste néanmoins lié aux contraintes génériques, et qui porte plus directement sur l'organisation phrastique, c'est-à-dire la structure thématique du texte.

\section{Structure thématique}

La structure ou progression thématique d'un texte concerne la répartition de l'information sur la ligne de la phrase. Une phrase progresse de son début - thème - vers sa fin - rhème - selon un dynamisme communicatif lié au statut pragmatique de l'ordre des mots dans la phrase (B. Combettes, 1983). Dans tout texte, il faut maintenir un équilibre entre les thèmes (ce dont on parle) qui sont les points de départ des énoncés, et les rhèmes (ce que l'on dit du thème) qui font progresser le texte.

L'ordre des éléments dans la phrase est fortement lié à la syntaxe, et ceci est surtout vrai de l'anglais et du français. Toutefois, ces langues permettent certaines latitudes, à travers par exemple l'emploi de la voix passive, des constructions emphatiques, la dislocation et d'autres procédés de la syntaxe marquée. Pour un non-natif, la tentation de respecter l'organisation canonique de la phrase en langue étrangère sera évidemment très forte. L'exemple suivant (tiré d'un projet scientifique de l'un de mes élèves) en est un cas typique.

In the picture there is an abundance of straight lines, particularly horizontal, so that frequencies are higher in the vertical domain. Coding techniques can take into account these characteristics.

21 Dans la première phrase, le thème est « the picture » et la partie rhématique de la phrase apporte des informations sur la composition de cette image. La deuxième phrase s'enchaîne sur un nouveau thème "coding techniques ». Par intuition, nous pressentons que le vrai thème de cette deuxième phrase est en fait les caractéristiques de l'image déjà évoquée. L'information pertinente, dans ce cas, est le fait que les "coding techniques» permettent de se rendre compte de ces divers traits. L'emploi de la diathèse passive aurait permis au scripteur de thématiser l'objet grammatical de cette phrase - «these characteristics» (ce dont il parle). Ce qui est, sur le plan de la communication, le plus important (et nouveau à cet instant) - «coding techniques» - aurait ainsi figuré en position de rhème.

These characteristics can be taken into account by the use of coding techniques 

fautes classiques d'orthographe) du point de vue du destinataire, les élèves seront ainsi amenés à réfléchir sur le discours en construction en tant que globalité. Dans un article paru dans Second Language Writing (1990), U. Connor et M. Farmer décrivent comment l'emploi d'une analyse de structure thématique peut être exploité en cours. Les étudiants doivent eux-mêmes repérer et souligner les thèmes des phrases dans un extrait de leur propre rédaction et en établir un schéma montrant les différents types de progression (progression à thème constant, progression linéaire...) qui s'y trouvent. L'enseignant les aide ensuite à interpréter les résultats et à proposer des améliorations dans l'organisation de leurs rédactions. Les implications de ce type d'exercice sur un plan théorique simple, ainsi que les possibilités d'auto-correction qui y sont préconisées, semblent très utiles. Nous passons beaucoup de temps à montrer aux élèves, par exemple, l'importance des mots-clés dans un paragraphe ou une section, mais si nous les incitons à analyser euxmêmes leurs écrits, simplement en soulignant les thèmes de phrases comme décrit cidessus, ils pourront ainsi mieux se rendre compte de certaines anomalies.

Tous les exercices de reformulation que nous proposons en cours peuvent aider les élèves à considérer un écrit en fonction d'un destinataire potentiel. En travaillant sur la progression thématique d'un texte et en réfléchissant sur le discours en construction au- 
delà de la phrase, ils comprennent à quel point on peut jongler avec la langue et ainsi faciliter l'accès au sens.

\section{Genre}

Cette prise en compte du destinataire par le scripteur doit être évaluée dans le cadre du " genre » dans lequel le texte se situe. Une fois que l'objet abstrait « texte » est replacé dans son contexte discursif, tout jugement sur son efficacité devra automatiquement inclure la question du genre. L'adéquation du texte à une situation de communication donnée, et ceci est surtout vraie dans le cadre de l'apprentissage, sera évaluée en termes de respect du type de texte exigé. Comme l'explique F. Rastier (1989 : 37) «Un genre est un programme de prescriptions positives ou négatives, et de licences qui règlent aussi bien la génération d'un texte que son interprétation; elles ne relèvent pas du système fonctionnel de la langue, mais d'autres normes sociales ». Les contraintes génériques correspondraient ainsi à une sorte de codification sociale et culturelle, inhérentes à la production et à l'interprétation de tout discours.

Dans quelle mesure est-il possible de modéliser les caractéristiques spécifiques au discours scientifique et, en l'occurrence, à l'anglais scientifique ? Est-ce que le discours scientifique constitue un genre en tant que tel ? Peu contesteraient la primauté de la mise en valeur de l'information à transmettre, d'où un style impersonnel. Ainsi pour Louis Trimble $(1985: 10)$

An EST(1) text is concerned only with the presentation of facts, hypotheses, and similar types of information. It is not concerned with the forms of written English that editorialize, express emotions or emotionally-based arguments or are fictional or poetic in nature.

Cependant, si ces prescriptions négatives nous permettent de cerner quelques caractéristiques du discours scientifique en général, elles ne permettent pas d'aborder les spécificités du discours scientifique dans des situations sociales distinctes.

La diversité des réalisations du discours scientifique peut être répertoriée selon des critères extra-linguistiques eux-mêmes très différents. G. Vigner (1979 : 107-108) propose une typologie fondée à la fois sur le public visé (discours didactique, discours heuristique [spécialistes], discours didactique vulgarisé), le degré d'organisation du texte, son support (revue, mémoire), l'étendue du domaine de référence et les actes mis en jeu (textes descriptifs, explicatifs, argumentatifs, etc.). Selon lui, le texte scientifique

est ainsi le produit d'un certain nombre de ces facteurs : pour accéder au sens il est nécessaire d'identifier ces composants, et de reconnaitre leurs dosages respectifs.

Tout programme d'apprentissage de la rédaction scientifique doit obligatoirement inclure une étude sur le type de textes auquel l'élève aura affaire. En ne retenant que le critère, par exemple, du public visé, nous relevons des objectifs de départ très différents. Si le but du discours heuristique est effectivement d'informer d'autres spécialistes, la priorité d'un discours didactique (un enseignant scientifique qui prépare un polycopié pour ses élèves) serait plutôt de former un public moins spécialisé. Quant au discours de vulgarisation, son objectif n'est-il pas, tout en informant, de distraire le grand public en rendant les informations scientifiques plus attrayantes? En fonction de ces objectifs de départ fort différents, il est logique de trouver non seulement un registre particulier mais des procédés rhétoriques eux-mêmes très diversifiés. 
Le registre approprié et les structures rhétoriques spécifiques à un type de texte donné devraient ainsi être abordés dans le contexte où ils apparaissent. À l'intérieur du type de texte visé, l'analyse permettra de repérer certaines récurrences linguistiques formelles. Toutefois, il ne s'agit pas d'apprendre des formulations standards définitives, car si l'objectif principal du discours scientifique est la transmission d'un savoir, encore faut-il que le scripteur puisse manipuler la langue qui véhicule ce savoir.

\section{Conclusion}

Dans cet exposé, j'ai essayé de cerner trois notions qui entrent en jeu dans l'interprétation de la cohérence d'un discours scientifique; la cohésion, la structure thématique et les contraintes génériques. Il ne s'agit pas de trois catégories d'interprétation distinctes, car elles sont, comme nous l'avons vu, largement interdépendantes et se côtoient à chaque étape de la mise en texte. Les éléments culturels sont présents à chaque niveau et viennent au premier plan dans toute discussion sur la notion de genre.

Pour le non-natif, la rédaction d'un document scientifique en anglais fait ressortir des considérations à la fois spécifiques au type d'exercice en question et également liées à sa compétence générale dans la langue. Les marques qui sont associées par convention à un certain type de texte doivent, de surcroît, être présentes dans les textes des apprenants. Les élèves qui suivent une formation scientifique ont généralement l'habitude de lire les documents relatifs à leur spécialité en anglais. C'est notre rôle de les familiariser avec les différents types de discours et de leur apprendre à en identifier les composants.

Malgré une certaine artificialité de la tâche en milieu scolaire, il faut rendre l'exercice le plus authentique possible. Si le scripteur destine son texte à un public précis, encore fautil que ces lecteurs puissent suivre l'organisation de l'écrit. La structure thématique du texte doit refléter le type de texte en question. Le scripteur ne doit jamais perdre de vue ces futurs lecteurs. Ce que recherche l'apprenant, c'est une maitrise linguistique qui lui permette de manipuler son texte dans un but rédactionnel précis. C'est le maniement de la langue tout court qui permet la réalisation de ces objectifs. Comme nous le rappellent T. Lebeaupin, C. Le Ninan, et Ph. Schmitt (1990 : 148)

pour qu'une langue puisse devenir langue de savoir, elle doit d'abord être capable de véhiculer du sens à quelqu'un.

\section{BIBLIOGRAPHIE}

Adam, J.M. 1985. Le texte narratif. Paris : Nathan.

Adam, J.M. 1977. « Ordre du texte, ordre du discours ». Pratiques 13.

Brown, G. et G. Yule. 1983. Discourse Analysis. Cambridge : Cambridge University Press.

Combettes, B. 1983. Pour une grammaire textuelle. Bruxelles : De Boeck. 
Combettes, B. et R. Tomassone. 1991. Le texte informatif. Bruxelles : De Boeck.

Charolles, M. 1978. « Introduction aux problèmes de la cohérence des textes ». Langue française 38.

Charolles, M. 1988. «Les études sur la cohérence, la cohésion et la connexité textuelle depuis la fin des années $1960 »$. Modèles linguistiques 10.

Connor, U et M. Farmer. 1990. «The teaching of topical structure analysis as a revision strategy for ESL writers ». In Kroll, B. (dir.), Second language writing. Cambridge : Cambridge University Press.

Fernandez, M. M. J. (dir.). 1987. « Traduction et vulgarisation scientifique ». Actes du $2^{e}$ Colloque Contrastif DISCOSS, LACITO-CNRS. Paris.

Fernandez, M. M. J. 1988. «L'analyse contrastive du discours. Bilan et devenir d'une approche pluridimensionnelle ». Modèles linguistiques 10.

François, F. 1980. «Linguistique et analyses de textes ». Linguistique (sous la direction de F. François). Paris : Presses Universitaires de France.

Halliday, M.A.K. et R. Hasan. 1976. Cohesion in English. Londres : Longman.

Huckin, Th. et A. Olsen. 1983. English for Science and Technology. New York : McGraw-Hill.

Lebeaupin, T. et al. 1990. «Enseigner la cohérence à des scientifiques étrangers ». Publics spécifiques et communication spécialisée, Hachette.

Rastier, F. 1989. Sens et textualité. Paris : Hachette.

Slatka, D. 1975. « L'ordre du texte ». Études de linguistique appliquée 19.

Trimble, L. 1985. English for Science and Technology. Cambridge : Cambridge University Press.

Thomas, S. et A, Kossoy. 1993. «L'incontournable dimension culturelle ». Actes de la $4^{e}$ Journée ERLA-GLAT, Brest.

Vigner, G. 1979. « Lire du texte au sens ». Didactique des langues étrangères. Paris : Hachette, CLE international.

\section{RÉSUMÉS}

Cet article porte sur trois notions qui entrent en jeu dans l'interprétation de la cohérence d'un discours, à savoir la cohésion, la structure thématique et les contraintes génériques. À partir de projets scientifiques rédigés dans le cadre d'un cours d'anglais scientifique à l'Institut National des Télécommunications, l'auteur essaye de cerner les éléments nécessaires à une communication réussie.

This article considers three concepts involved in gauging the degree of coherence of written discourse: textual cohesion, topical structure analysis and genre constraints. Basing my observations on scientific projects written by French engineering students as part of the course requirement in an ESP programme at the Institut National des Télécommunications, I attempt to determine what makes a successful project. 
INDEX

Mots-clés : cohérence, cohésion, culture, discours scientifique, genre, structure thématique

Keywords : coherence, cohesion, culture, genre, scientific discourse, topical structure analysis

\section{AUTEUR}

\section{SHIRLEY CARTER-THOMAS}

Shirley Carter-Thomas enseigne à l'Institut National des Télécommunications à Évry. shirley.thomas@telecom-em.eu 